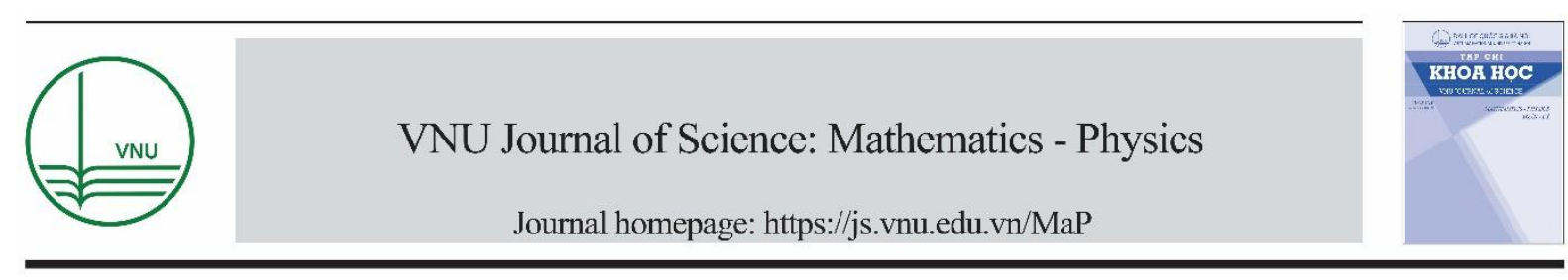

Original Article

\title{
Prediction of the Higgs and Top Quark Masses by Discrete Dimensions Revisited
}

\author{
Nguyen Van Dat ${ }^{2, *}$, Nguyen Ai Viet ${ }^{1,2}$, Pham Tien $\mathrm{Du}^{3}$ \\ ${ }^{I}$ VNU Information Technology Institute, Vietnam National University, Hanoi, \\ 144 Xuan Thuy, Hanoi, Vietnam \\ ${ }^{2}$ Faculty of Physics, VNU University of Science, Vietnam National University, Hanoi, \\ 334 Nguyen Trai, Hanoi, Vietnam \\ ${ }^{3}$ Faculty of Physics, Thuy Loi University, 175 Tay Son, Hanoi, Vietnam
}

Received 20 November 2019, Accepted 02 March 2020

\begin{abstract}
A new metric structure of the discretized Kaluza-Klein theory can give us new knowledge about extra-dimension. It can provide the new predictions of the top quark and Higgs mass that studied by Viet $[15,16]$ in another model. Compare the results of two approaches we can see that the new model is more agreement with experimental data.
\end{abstract}

Keywords: Higgs mass, top quark mass, discretized Kaluza-Klein theory, DKKT.

\section{Introduction}

In physics, predictions usually are based on the differential equations. The profound essence of those is the derivative, which relates the value of a quantity at a given point to the one in its infinitesimal neighborhood. One of most important theoretical construction tool for unified theories is the multi-dimensional space-time. The extra dimension introduces a new variable to relate different physical theories. The derivative in the additional dimension can lead to new predictions. One example is the Kaluza-Klein theory, which unifies gravity and electromagnetism [1,2]. This theoretical framework can predict massive modes, charge quantization and interesting physical relations. Today, this idea is already a de facto standard in physics to construct the unified theories such as string, supergravity, super Yang-Mills ones.

\footnotetext{
* Corresponding author.

Email address: dnvdat@gmail.com

https//doi.org/ 10.25073/2588-1124/vnumap.4435
} 
The compact continuous extra dimension with the continuous variable derivative is very powerful in predictions. However, it suffers a serious drawback since it implies infinite towers of massive modes, which are Fourier coefficients in an expansion of the extended fields. The infinite number of massive modes can cause a lot of theoretical and observational inconsistencies.

To avoid this shortcoming, some authors, in particular Viet and Wali [3-6] have promoted the idea of discrete extra dimensions. In fact, the extended Hilbert-Einstein-Cartan theory with discrete extra dimensions can contain gravity and nonabelian gauge theories paving the way to a unified description of all interactions $[7,8]$. On the other hand, the gauge theory with one discrete dimension can naturally lead to the existence of the Higgs field with a quartic potential, triggering the spontaneous gauge symmetry breaking to give mass to the quark-leptons and gauge fields. Therefore, one can have a theory with two discrete extra dimensions to unified all the interactions and the Higgs field as components of the extended gravity [9] with a finite field content. In such a framework, there is no difference between the constructions of the Einstein and Yang-Mills theories. More recently, the idea of discrete dimension has also been explored by other authors [10-12].

The Viet-Wali's framework originally has been formulated on the mathematically rigorous foundation of noncommutative geometry a la Connes [13]. However, in the present form, it becomes an independent procedure based one the discrete derivatives and in a better parallelism with the existing physical theories. Thus, DKKT can provide much more physical insights, allowing to overcome the no-go theorem by Chamseddine, Felder and Frohlich [14] to include vector field in the extended gravity paving the way to unfify gravity with other gauge interactions.

In the discrete dimensional models, the usual derivatives are replaced by the discrete ones, which are finite differences, which are defined in terms of mass parameters, which are inverse of the distance between the discrete points. Therefore, these frameworks have a strong predictive powers. Viet has also explored this property of the discrete dimension to imply phenomenological predictions, in particular the Weinberg angle, Higgs and top quark masses $[15,16]$. The prediction of top quark mass was made shortly before its experimental discovery with a surprising agreement. On the other hand, since the Higgs mass was not known at that time, the predictions of it were $241 \mathrm{GeV}$ and $350 \mathrm{GeV}$ with certain choices of the metrics.

In the Standard Model, the Higgs potential is given with two parameters. Therefore the Higgs mass and its vacuum expectation value (VEV) are free parameters. The masses of quarks and leptons are products of the Higgs' VEV and the diagonalized Yukawa coupling constants. Therefore, quark and lepton masses are also free parameters of the Standard Model.

In the extended gauge theory with a discrete dimension consisting of two points, all these parameters have to satisfy some constraints leading to predictions. In the previous paper [17], we have discussed the prediction of the Weinberg angle in the gauge theory extended by a discrete dimension. Surprisingly, this framework has given the value $\sin ^{2} \theta_{w}=0.23077$ in a very good agreement with experiments. One can imply that the space-time structure at the electroweak energy scale is compatible with the one extended with a discrete dimension.

In this paper, we reexamine the predictions of the Higgs and top quark masses of Viet $[15,16]$. We will include into the framework the new Dirac operator introduced in [8]. The new metric will be used to give a better fit to the Higgs mass. This idea has been used in a low energy application [18]. Lastly, in the papers $[15,16]$ Viet has developed a modified formulation, where the discrete dimension is described by a pair of hermitic conjugate variables $Z$ and $\bar{Z}$. This formalism can help to avoid a strange extension of the wedge product to $d x^{5} \wedge d x^{5} \neq 0$. However, this leads to a new mathematical framework, which is not in a direct parallelism and familiar to physicists. So we choose just one 
discrete variable keeping in mind that, it is an equivalent way to introduce the Higgs potential, even if it has a less natural wedge product.

\section{Differential Calculus and the Metric Structure}

The differential calculus with a discrete derivative can be formulated in a perfect parallelism with the ordinary one [3-6, 8]. Let us overview the most important formulas to be used in this paper.

The discrete dimension of two points implies that the space-time has two sheets. We follow the Connes-Lott model [13] to postulate that these two copies of space-time are where the chiral quarkleptons exist. Therefore, the right-handed chiral quark-leptons are Kaluza-Klein partner of the left ones. Therefore, one can represent the Kaluza-Klein pairs as the following 2-colummn spinor.

$$
\Psi=\left[\begin{array}{l}
\psi_{L} \\
\psi_{R}
\end{array}\right] .
$$

Note that $\psi_{L}$ and $\psi_{R}$ do not necessarily have the same internal properties. They might have also the generation, isospin, color, quark or lepton indexes.

The second element is the Algebra using for function operations $\mathcal{A}=\mathcal{A}_{L} \oplus \mathcal{A}_{R}$, where $\mathcal{A}_{I}=\mathcal{C}^{\infty}\left(\mathcal{M}^{4}\right), I=L, R$. The elements of this algebra is represented by 0 -form diagonal matrix $F$,

$$
F(x)=\left[\begin{array}{ll}
f_{L}(x) & 0 \\
0 & f_{R}(x)
\end{array}\right] .
$$

The third element is the Dirac operator, which that can be defined as an extension of the normal Dirac operator as $D=d . \mathbf{e}+\Theta$, where $d=\gamma^{\mu} \partial_{\mu}$ is usual Dirac operator in the four dimensions spacetime $\mathcal{M}^{4}$

$$
D=\left[\begin{array}{ll}
\gamma^{\mu} \partial_{\mu} & -i m \gamma^{5} / \lambda^{2} \\
i m \gamma^{5} / \lambda^{2} & \gamma^{\mu} \partial_{\mu}
\end{array}\right], \Theta=\left[\begin{array}{ll}
0 & -i m \gamma^{5} / \lambda^{2} \\
i m \gamma^{5} / \lambda^{2} & 0
\end{array}\right] .
$$

With NCG space-time defined with the above spectral triplet, we can calculate the derivative of the 0 -forms by acting the Dirac operator on function $\mathrm{F}$ as follows

$$
D F=[D, F]=\left[\begin{array}{ll}
\gamma^{\mu} \partial_{\mu} f_{L}(x) & i m \gamma^{5}\left(f_{L}(x)-f_{R}(x)\right) / \lambda^{2} \\
i m \gamma^{5}\left(f_{L}(x)-f_{R}(x)\right) / \lambda^{2} & \gamma^{\mu} \partial_{\mu} f_{R}(x)
\end{array}\right]
$$

We can rewrite it in the following form

$$
D F=D X^{\mu}\left[D_{\mu}, F\right]+D X^{5} \sigma^{\dagger}\left[D_{5}, F\right]=D X^{\mu} \partial_{\mu} F+D X^{5} \sigma^{\dagger} \partial_{5} F,
$$

Where

$$
D_{5}=\left[\begin{array}{ll}
0 & -m \\
m & 0
\end{array}\right], \sigma^{\dagger}=\left[\begin{array}{ll}
0 & -1 \\
1 & 0
\end{array}\right] .
$$

If we use the representation of Dirac matrix, $D X^{\mu}$ can be replaced by the generalized $\gamma$-matrices 


$$
\Gamma^{\mu}=\left[\begin{array}{cc}
\gamma^{\mu} & 0 \\
0 & \gamma^{\mu}
\end{array}\right], \Gamma^{5}=\left[\begin{array}{cc}
0 & i \gamma^{5} / \lambda^{2} \\
-i \gamma^{5} / \lambda^{2} & 0
\end{array}\right]
$$

The metric can be defined in terms of the generalized Dirac matrices $\Gamma^{M}$ as

$$
\left\{\Gamma^{M}, \Gamma^{N}\right\}=2 G^{M N}=\operatorname{diag}\left(-1,1,1,1,1 / \lambda^{4}\right) .
$$

That is to say the external metric is flat, while the internal one is non-trivial, to be determined by a scale parameter $\lambda^{2}$. This metric has been used recently in [18] to study the discrete dimension at low energy.

In the general case, the metric can be defined by introducing the scalar product of the differential elements as follows

$$
\begin{aligned}
& \left\langle D X^{M}, D X^{N}\right\rangle=G^{M N}=\operatorname{diag}\left(-1,1,1,1,1 / \lambda^{4}\right) \\
& G_{M N}=\operatorname{diag}\left(-1,1,1,1, \lambda^{4}\right), \quad \operatorname{det} G=-\lambda^{4} .
\end{aligned}
$$

Since the metric is not trivial, according to the General Relativity, the Lagrangian must have an additional factor of $\sqrt{-\operatorname{det} G}=\lambda^{2}$.

Since the derivative of a 0 -form is a 1 -form, we can extend the module of 1 -forms, which is the generalization of the vector field in NCG to the following form

$$
U=\Gamma^{M} U_{M}=\Gamma^{\mu} U_{\mu}+\Gamma^{5} U_{5}=\left[\begin{array}{ll}
\gamma^{\mu} u_{\mu L} & i \gamma^{5} u_{5 R} / \lambda^{2} \\
-i \gamma^{5} u_{5 L} / \lambda^{2} & \gamma^{\mu} u_{\mu R}
\end{array}\right]
$$

where $U_{M}$ is generalized functions ( 0 -forms). The 1-form $U$ contains two vectors and two scalars.

Now we can define the 2-form to be used as generalized field strength or curvature. The 2-forms must extend from the derivatives of 1 -forms. We have to define wedge product of two 1-forms as follows

$$
\begin{gathered}
D X^{\mu} \wedge D X^{v}=-D X^{v} \wedge D X^{\mu}, D X^{\mu} \wedge D X^{5}=-D X^{5} \wedge D X^{\mu}, D X^{5} \wedge D X^{5} \neq 0, \\
U \wedge V=D X^{M} \wedge D X^{N}(U \wedge V)_{M N},(U \wedge V)_{\mu \nu}=-(U \wedge V)_{v \mu}=\frac{1}{2}\left(U_{\mu} V_{v}-U_{v} V_{\mu}\right) \\
(U \wedge V)_{\mu 5}=-(U \wedge V)_{5 \mu}=\frac{1}{2}\left(\tilde{U}_{\mu} V_{5}-U_{5} V_{\mu}\right),(U \wedge V)_{55}=\tilde{U}_{5} V_{5}
\end{gathered}
$$

where tidle operation " $\sim$ " on a generalized function is defined as follows

$$
\tilde{F}=f_{+} \mathbf{e}-f_{-} \mathbf{r}, f_{ \pm}=\frac{1}{2}\left(f_{1} \pm f_{2}\right)
$$

where

$$
\mathbf{e}=\left[\begin{array}{ll}
1 & 0 \\
0 & 1
\end{array}\right], \mathbf{r}=\left[\begin{array}{cc}
1 & 0 \\
0 & -1
\end{array}\right]
$$

Exterior derivative of 1 -forms is given as

$$
\begin{aligned}
D U & =[D, U]=D X^{M} \wedge D X^{N}(D U)_{M N} \\
(D U)_{\mu v} & =\frac{1}{2}\left(\partial_{\mu} U_{v}-\partial_{\nu} U_{\mu}\right)=-(D U)_{v \mu} \\
(D U)_{\mu 5} & =\frac{1}{2}\left(\partial_{\mu} U_{5}-m\left(U_{\mu}-\tilde{U}_{\mu}\right)\right)=-(D U)_{5 \mu} \\
(D U)_{55} & =m\left(U_{5 L}+U_{5 R}\right)
\end{aligned}
$$


The scalar product of two 2-forms is defined via the metric in the following formulas

$$
\begin{aligned}
& <D X^{M} \wedge D X^{N}, D X^{P} \wedge D X^{Q}>=G^{M P} G^{N Q}-G^{M Q} G^{N P} \\
& <X, Y>=X_{M N}^{\dagger}<D X^{M} \wedge D X^{N}, D X^{P} \wedge D X^{Q}>Y_{P Q}
\end{aligned}
$$

\section{The Extended Gauge Sector Coupled to Quark-leptons}

The gauge field A in DKKT takes the following form

$$
A=\left(\begin{array}{ll}
\gamma^{\mu} a_{\mu L} & i \gamma^{5} a_{5}^{\dagger} \\
-i \gamma^{5} a_{5} & \gamma^{\mu} a_{\mu R}
\end{array}\right)=\Gamma^{\mu} A_{\mu}+\Gamma^{5} A_{5}=D X^{\mu} A_{\mu}+D X^{5} A_{5} .
$$

So, the gauge fields $a_{L}, a_{R}$ and complex scalar field $a_{5}$ are choosen as elements of the following $2 \times 2$ matrices

$$
A_{\mu}=\left(\begin{array}{ll}
a_{L \mu} & 0 \\
0 & a_{R \mu}
\end{array}\right), A_{5}=\left(\begin{array}{ll}
a_{5} & 0 \\
0 & a_{5}^{\dagger}
\end{array}\right) .
$$

Let us specialized to the case of the Standard Model with the following quark-leptons

Focusing on quark and lepton families including the right-handed neutrino, we have the following left- and right-handed chiral quark-lepton representation,

$$
\psi_{L A}=\left[\begin{array}{c}
q_{L}^{c} \\
l_{L}
\end{array}\right]_{A}, \psi_{R A}=\left[\begin{array}{c}
u_{R}^{c} \\
d_{R}^{c} \\
e_{R} \\
v_{R}
\end{array}\right]_{A},
$$

where $\mathrm{c}=1,2,3$ is the color index, $A$ is the family index, the number of which we leave arbitrary. Note that $e_{A}, v_{A}, A=1,2,3$ represents respectively the electron, muon and tau and their neutrinos, while $u_{A}, d_{A}$ does the $\mathrm{u}-, \mathrm{c}-, \mathrm{t}$-quarks and d-, s-, b-ones.

The gauge sector with the usual physical gauge $W_{\mu}(x), B_{\mu}(x)$ and Higgs $h(x)$ fields as follows

$$
\begin{aligned}
& a_{L \mu}=g W_{\mu}^{a}(x) \frac{\tau^{a}}{2} \otimes \mathbf{1}_{4} \otimes \mathbf{1}_{N_{F}}-g^{\prime} \frac{Y_{L}}{2} B_{\mu}(x) \otimes \mathbf{1}_{2} \otimes \mathbf{1}_{N_{F}} \\
& a_{R \mu}=-g^{\prime} \frac{Y_{R}}{2} B_{\mu}(x) \otimes \mathbf{1}_{N_{F}} \\
& a_{5}=f_{k}\left[\begin{array}{ll}
h_{0}-\frac{m}{f_{k}} & h_{1}^{*} \\
-h_{1} & h_{0}^{*}-\frac{m}{f_{k}}
\end{array}\right] \otimes \mathrm{K}, H=\sqrt{2}\left[\begin{array}{l}
h_{0} \\
h_{1}
\end{array}\right] .
\end{aligned}
$$

where $Y_{L, R}$ are the hypercharge operators acting on the left and right-handed chiral quark-leptons. Since the gauge fields $a_{L, R \mu}$ are operators acting on the chiral quark-leptons, we must have include the corresponding unit matrices in Eqs.(21). $g, g$ ' and $f_{\kappa}$ are parameters, $H$ is the usual Higgs doublet. 
The hyper charge operators are the same for all the families and hence we specify them to be

$$
\begin{aligned}
Y_{L} & =\left[\begin{array}{cc}
\frac{1}{3} \mathbf{1}_{3} & 0 \\
0 & -1
\end{array}\right], \\
Y_{R} & =\left[\begin{array}{cccc}
\frac{4}{3} \mathbf{1}_{3} & 0 & 0 & 0 \\
0 & -\frac{2}{3} \mathbf{1}_{3} & 0 & 0 \\
0 & 0 & -2 & 0 \\
0 & 0 & 0 & 0
\end{array}\right] .
\end{aligned}
$$

In Eqs.(21), $K$ is the matrix of Yukawa coupling constants. In fact, the coupling of quark-leptons represented in Eqs.(20) to the extended gauge sector in our model is given in the compact form

$$
\begin{gathered}
\mathcal{L}_{f-g}=i \bar{\Psi}(D-i A) \Psi=\mathcal{L}_{g-f}(L)+\mathcal{L}_{g-f}(R)+\mathcal{L}_{H-f} \\
\mathcal{L}_{g-f}(L, R)=i \bar{\psi}_{L, R} \gamma^{\mu}\left(\partial_{\mu}-i a_{\mu L}\right) \psi_{L, R} \\
\mathcal{L}_{H-f}=-\frac{1}{\lambda^{2}}\left(\bar{\psi}_{L}\left(m+a_{5}\right) \psi_{R}+\bar{\psi}_{R}\left(m+a_{5}^{\dagger}\right) \psi_{L}\right) .
\end{gathered}
$$

The factor $\sqrt{-\operatorname{det} G}=\lambda^{2}$ in the above Lagrangians has been absorbed into the fermions by redefinition $\Psi^{\prime}=\lambda \Psi$. We have also omitted the "prime" after redefinition hopefully without confusion.

The Yukawa coupling of the Higgs field $H$ to quark-leptons now emerges naturally as a part of the extended gauge-quark-lepton interaction. Hence, there are some constraints on the model parameters leading to predictions as we will be in this paper. Let us examine the Yukawa Lagrangian $L_{H-f}$ in more details to see the physical content of the matrix $K . L_{H-f}$ can be separated into the Yukawa coupling to quark, electrons and neutrino as follows

$$
\mathcal{L}_{H-f}=-\frac{f_{\kappa}}{\lambda^{2}}\left(\bar{\psi}_{L} H \otimes K \psi_{R}+\bar{\psi}_{R} K^{\dagger} \otimes \bar{H} \psi_{L}\right)=\mathcal{L}_{H-u}+\mathcal{L}_{H-d}+\mathcal{L}_{H-e}+\mathcal{L}_{H-v},
$$

where

$$
\begin{aligned}
& \mathcal{L}_{H-u}=-\frac{f_{\kappa}}{\lambda^{2}} K_{A B}^{u}\left(\left(\bar{u}_{L}^{c} h_{0}+\bar{d}_{L}^{c} h_{1}\right)_{A} u_{R B}^{c}+\bar{u}_{R A}^{c}\left(\left(\bar{h}_{0} u_{L}^{c}+\bar{h}_{1} d_{L}^{c}\right)_{B}\right)\right) \\
& \mathcal{L}_{H-d}=-\frac{f_{\kappa}}{\lambda^{2}} K_{A B}^{u}\left(\left(\bar{u}_{L}^{c} h_{0}+\bar{d}_{L}^{c} h_{1}\right)_{A} d_{R B}^{c}+\bar{d}_{R A}^{c}\left(\left(\bar{h}_{0} u_{L}^{c}+\bar{h}_{1} d_{L}^{c}\right)_{B}\right)\right) \\
& \mathcal{L}_{H-e}=-\frac{f_{\kappa}}{\lambda^{2}} K_{A B}^{u}\left(\left(\bar{e}_{L}^{c} h_{0}+\bar{v}_{L}^{c} h_{1}\right)_{A} e_{R B}^{c}+\bar{e}_{R A}^{c}\left(\left(\bar{h}_{0} e_{L}^{c}+\bar{h}_{1} v_{L}^{c}\right)_{B}\right)\right) \\
& \mathcal{L}_{H-v}=-\frac{f_{\kappa}}{\lambda^{2}} K_{A B}^{u}\left(\left(\bar{e}_{L}^{c} h_{0}+\bar{v}_{L}^{c} h_{1}\right)_{A} v_{R B}^{c}+\bar{v}_{R A}^{c}\left(\left(\bar{h}_{0} e_{L}^{c}+\bar{h}_{1} v_{L}^{c}\right)_{B}\right)\right) .
\end{aligned}
$$


Therefore, the usual Yukawa coupling constants is related to the elements of the matrix $K$ as follows

$$
\begin{aligned}
& \left(f_{u}\right)_{A B}=-\frac{f_{\kappa}}{\lambda^{2}} K_{A B}^{u} \\
& \left(f_{d}\right)_{A B}=-\frac{f_{\kappa}}{\lambda^{2}} K_{A B}^{d} \\
& \left(f_{e}\right)_{A B}=-\frac{f_{\kappa}}{\lambda^{2}} K_{A B}^{e} \\
& \left(f_{v}\right)_{A B}=-\frac{f_{\kappa}}{\lambda^{2}} K_{A B}^{v} .
\end{aligned}
$$

All other elements of the matrix $K$ vanish. That is to say, the matrix $K$ mixes the quarks and leptons of the same type (including also the color) between the generations. This leads to the CKS and PNMS flavor mixing matrices.

Since the trace is invariant under the unitary transformation we can see that, after diagonalizing the Yukawa coupling matrix by mixing quark-lepton gauge eigenstates to obtain the mass ones, the following trace formula holds

$$
v \frac{f_{\kappa}}{\lambda^{2}} \operatorname{Tr}\left(K^{\dagger} K\right)=\sum m_{i}^{2} \sim m_{t}^{2},
$$

where the sum is over all the quark-lepton types, which can be approximated by the top quark mass square. We will use this relation later as a constraint.

Now we are ready to construct the Lagrangian for the extended gauge sector. The field strength is defined through wedge product and derivative of gauge fields as follows

$$
F=D A+A \wedge A \text {. }
$$

We can calculate its components by using the following formula

$$
\begin{aligned}
F & =D X^{\mu} \wedge D X^{v} \frac{1}{2}\left(\partial_{\mu} A_{v}-\partial_{v} A_{\mu}+\left[A_{\mu}, A_{v}\right]\right) \\
& +2 D X^{\mu} \wedge D X^{5} \frac{1}{2}\left(\partial_{\mu}+\left(\tilde{A}_{\mu}-A_{\mu}\right)\right)\left(A_{5}+m\right)+D X^{5} \wedge D X^{5}\left(m\left(a_{5}+a_{5}^{\dagger}\right)+a_{5}^{+} a_{5}\right) \\
& =D X^{\mu} \wedge D X^{v} F_{\mu \nu}+2 D X^{\mu} \wedge D X^{5} F_{\mu 5}+D X^{5} \wedge D X^{5} F_{55}
\end{aligned}
$$

Let us calculate explicitly each component of the field strength 2-form in terms of the physical boson and Higgs fields. The first component can be calculated as follow

$$
\begin{aligned}
F_{\mu \nu} & =\frac{1}{2}\left(\partial_{\mu} A_{\nu}-\partial_{\nu} A_{\mu}+\left[A_{\mu}, A_{v}\right]\right)=f_{\mu \nu L}+f_{\mu \nu R} \\
& =\frac{1}{2}\left(\partial_{\mu} a_{\nu L}-\partial_{\nu} a_{\mu L}+\left[a_{\mu L}, a_{v L}\right]\right)+\frac{1}{2}\left(\partial_{\mu} a_{\nu R}-\partial_{\nu} a_{\mu R}+\left[a_{\mu R}, a_{v R}\right]\right) \\
& =\left(\frac{\mathrm{g}}{4} W_{\mu \nu} \otimes \mathbf{1}_{4}-g^{\prime} \frac{\mathrm{Y}_{\mathrm{L}} \otimes \mathbf{1}_{2}+\mathrm{Y}_{\mathrm{R}}}{4} \mathrm{~B}_{\mu \nu}\right) \otimes \mathbf{1}_{\mathrm{N}_{f}}
\end{aligned}
$$

where 


$$
W_{\mu \nu}=\tau^{a} W_{\mu \nu}^{a}=\tau^{a}\left(\partial_{\mu} W_{\nu}^{a}-\partial_{\nu} W_{\mu}^{a}+\frac{g}{2} f_{a b c}\left[W_{\mu}^{b}, W_{v}^{c}\right]\right), B_{\mu \nu}=\partial_{\mu} B_{v}-\partial_{\nu} B_{\mu} .
$$

The gauge fields do not mix the quark-lepton generation. We omit the gluon fields as they do not influence the predictions made in this paper.

The second component of the field strength tensor is calculated as follows

$$
F_{\mu 5}=\frac{1}{2}\left(\partial_{\mu}+\left(\tilde{A}_{\mu}-A_{\mu}\right)\right)\left(A_{5}+m\right)=f_{\mu 5 L}+f_{\mu 5 R}
$$

where $f_{\mu 5 L}, f_{\mu 5 R}$ is

$$
\begin{aligned}
f_{\mu 5 L}= & \frac{1}{2}\left(\partial_{\mu}+\left(a_{\mu R}-a_{\mu L}\right)\right)\left(a_{5}+m\right)=\frac{1}{2} f_{k}\left(\left[\begin{array}{cc}
\partial_{\mu} h_{0} & \partial_{\mu} h_{1}^{*} \\
-\partial_{\mu} h_{1} & \partial_{\mu} h_{0}^{*}
\end{array}\right]\right. \\
& \left.+\left[\begin{array}{cc}
h_{0} & h_{1}^{*} \\
-h_{1} & h_{0}^{*}
\end{array}\right]\left(-g W_{\mu}^{a}(x) \frac{\tau^{a}}{2} \otimes \mathbf{1}_{4}+g^{\prime} \frac{Y_{L} \otimes \mathbf{1}_{2}-Y_{R}}{2} B_{\mu}(x)\right)\right) \otimes K \\
f_{\mu 5 R}= & \frac{1}{2}\left(\partial_{\mu}-\left(a_{\mu R}-a_{\mu L}\right)\right)\left(a_{5}^{\dagger}+m\right)=\frac{1}{2} f_{k}\left(\left[\begin{array}{cc}
\partial_{\mu} h_{0}^{*} & -\partial_{\mu} h_{1}^{*} \\
\partial_{\mu} h_{1} & \partial_{\mu} h_{0}
\end{array}\right]\right. \\
& \left.-\left[\begin{array}{cc}
h_{0}^{*} & -h_{1}^{*} \\
h_{1} & h_{0}
\end{array}\right]\left(-g W_{\mu}^{a}(x) \frac{\tau^{a}}{2} \otimes \mathbf{1}_{4}+g^{\prime} \frac{Y_{L} \otimes \mathbf{1}_{2}-Y_{R}}{2} B_{\mu}(x)\right)\right) \otimes K^{\dagger}
\end{aligned}
$$

The third component of the field strength tensor is calculated as follow

$$
\begin{aligned}
F_{55} & =m\left(a_{5}^{\dagger}+a_{5}\right)+a_{5}^{\dagger} a_{5} \\
& =m\left(f_{k}\left(h_{0}^{*}+h_{0}\right)-2 m\right)+\left(f_{k} h_{0}^{*}-m\right)\left(f_{k} h_{0}-m\right)+f_{k}^{2} h_{1}^{*} h_{1} \\
& =f_{k}^{2}\left(\left(h_{0}^{*} h_{0}+h_{1}^{*} h_{1}\right)-\frac{m^{2}}{f_{k}^{2}}\right)=\frac{f_{k}^{2}}{2}\left(\bar{H} H-2 \frac{m^{2}}{f_{k}^{2}}\right) \otimes K^{\dagger} K
\end{aligned}
$$

The Lagrangian of the gauge sector now is calculated as

$$
L_{g}=-\frac{\lambda^{2}}{f_{\kappa}^{2}}<F, F>=-\frac{\lambda^{2}}{f_{\kappa}^{2}} \operatorname{Tr}\left(F_{\mu \nu}^{\dagger} F^{\mu \nu}-\frac{2}{\lambda^{4}} F_{\mu 5}^{\dagger} F_{5}^{\mu}+\frac{1}{\lambda^{8}} F_{55}^{\dagger} F_{55}\right) .
$$

assuming that $G^{M N}=\operatorname{diag}\left(-1,1,1,1,1 / \lambda^{4}\right)$. The factor $\lambda^{2}$ is due to the non trivial metric via $\sqrt{-\operatorname{det} G}$.

Let calculate the first term in the Lagrangian, we have

$$
F_{\mu \nu}^{\dagger} F^{\mu \nu}=f_{\mu \nu L}^{\dagger} f_{L}^{\mu \nu}+f_{\mu \nu R}^{\dagger} f_{R}^{\mu \nu}=\left(\frac{g^{2}}{16} W_{\mu \nu} W^{\mu \nu} \otimes 1_{4}+g^{\prime 2} \frac{\mathrm{Y}_{\mathrm{L}}^{2} \otimes 1_{2}+\mathrm{Y}_{\mathrm{R}}^{2}}{16} \mathrm{~B}_{\mu \nu} \mathrm{B}^{\mu \nu}\right) \otimes \mathbf{1}_{\mathrm{N}_{f}}
$$

so we can take the trace of Eq.(45) to obtain 


$$
F_{\mu \nu}^{\dagger} F^{\mu \nu}=f_{\mu \nu L}^{\dagger} f_{L}^{\mu \nu}+f_{\mu \nu R}^{\dagger} f_{R}^{\mu \nu}=\left(\frac{g^{2}}{16} W_{\mu \nu} W^{\mu \nu} \otimes 1_{4}+g^{\prime 2} \frac{\mathrm{Y}_{\mathrm{L}}^{2} \otimes 1_{2}+\mathrm{Y}_{\mathrm{R}}^{2}}{16} \mathrm{~B}_{\mu \nu} \mathrm{B}^{\mu \nu}\right) \otimes \mathbf{1}_{\mathrm{N}_{f}}
$$

The second term can be derived similarly

$$
\begin{aligned}
F_{\mu 5}^{\dagger} F^{\mu 5}= & \left(f_{\mu 5 L}^{\dagger}+f_{\mu 5 R}^{\dagger}\right)\left(f^{\mu 5 L}+f^{\mu 5 R}\right)=f_{\mu 5 L}^{\dagger} f^{\mu 5 L}+f_{\mu 5 R}^{\dagger} f^{\mu 5 R} \\
f_{\mu 5 L}^{\dagger} f^{\mu 5 L}= & \frac{1}{2 \lambda^{4}}\left(\partial_{\mu}+\left(a_{\mu R}-a_{\mu L}\right)\right)\left(a_{5}^{\dagger}+m\right) \frac{1}{2}\left(\partial^{\mu}+\left(a_{R}^{\mu}-a_{L}^{\mu}\right)\right)\left(a_{5}+m\right) \\
= & \frac{1}{4 \lambda^{4}}\left(\partial_{\mu} a_{5}^{\dagger} \partial^{\mu} a_{5}+\partial_{\mu} a_{5}^{\dagger}\left(a_{5}+m\right)\left(a_{R}^{\mu}-a_{L}^{\mu}\right)+\partial^{\mu} a_{5}\left(a_{5}^{\dagger}+m\right)\left(a_{\mu R}-a_{\mu L}\right)\right. \\
& \left.+\left(a_{5}^{\dagger}+m\right)\left(a_{5}+m\right)\left(a_{\mu R}-a_{\mu L}\right)\left(a_{R}^{\mu}-a_{L}^{\mu}\right)\right) \\
f_{\mu 5 R}^{\dagger} f^{\mu 5 R}= & \frac{1}{2 \lambda^{4}}\left(\partial_{\mu}-\left(a_{\mu R}-a_{\mu L}\right)\right)\left(a_{5}+m\right) \frac{1}{2}\left(\partial^{\mu}-\left(a_{R}^{\mu}-a_{L}^{\mu}\right)\right)\left(a_{5}^{\dagger}+m\right) \\
= & \frac{1}{4 \lambda^{4}}\left(\partial_{\mu} a_{5} \partial^{\mu} a_{5}^{\dagger}-\partial_{\mu} a_{5}\left(a_{5}^{\dagger}+m\right)\left(a_{R}^{\mu}-a_{L}^{\mu}\right)-\partial^{\mu} a_{5}^{\dagger}\left(a_{5}+m\right)\left(a_{\mu R}-a_{\mu L}\right)\right. \\
& \left.+\left(a_{5}+m\right)\left(a_{5}^{\dagger}+m\right)\left(a_{\mu R}-a_{\mu L}\right)\left(a_{R}^{\mu}-a_{L}^{\mu}\right)\right)
\end{aligned}
$$

so

$$
\begin{aligned}
& \operatorname{Tr}\left(F_{\mu 5}^{\dagger} F^{\mu 5}\right)=\operatorname{Trace}\left(f_{\mu 5 L}^{\dagger} f^{\mu 5 L}+f_{\mu 5 R}^{\dagger} f^{\mu 5 R}\right) \\
& =\frac{1}{2 \lambda^{4}} \operatorname{Tr}\left(\partial_{\mu} a_{5}^{\dagger} \partial^{\mu} a_{5}+\left(a_{5}^{\dagger}+m\right)\left(a_{5}+m\right)\left(a_{\mu R}-a_{\mu L}\right)\left(a_{R}^{\mu}-a_{L}^{\mu}\right)\right) \\
& =\frac{1}{2 \lambda^{4}} \operatorname{Tr}\left(K^{\dagger} K\right) \operatorname{Tr}\left(f_{k}^{2}\left[\begin{array}{cc}
\partial_{\mu} h_{0}^{*} \partial^{\mu} h_{0}+\partial_{\mu} h_{1}^{*} \partial^{\mu} h_{1} & 0 \\
0 & \partial_{\mu} h_{0}^{*} \partial^{\mu} h_{0}+\partial_{\mu} h_{1}^{*} \partial^{\mu} h_{1}
\end{array}\right]\right. \\
& \left.+f_{k}^{2}\left[\begin{array}{cc}
h_{0} h_{0}^{*}+h_{1} h_{1}^{*} & 0 \\
0 & h_{0} h_{0}^{*}+h_{1} h_{1}^{*}
\end{array}\right]\left(g^{2} W_{\mu}^{a} W^{\mu b} \frac{\tau^{a} \tau^{b}}{4} \otimes \mathbf{1}_{4}+g^{\prime 2} \frac{Y_{L}^{2} \otimes \mathbf{1}_{2}+Y_{R}^{2}}{4} B_{\mu} B^{\mu}\right)\right) \\
& =\frac{\operatorname{Tr}\left(K^{\dagger} K\right)}{2 \lambda^{4}}\left(2 f_{k}^{2}\left(\partial_{\mu} h_{0}^{*} \partial^{\mu} h_{0}+\partial_{\mu} h_{1}^{*} \partial^{\mu} h_{1}\right)+f_{k}^{2} 2\left(h_{0} h_{0}^{*}+h_{1} h_{1}^{*}\right)\left(2 g^{2} W_{\mu}^{a} W^{\mu a}+g^{\prime 2} \frac{5}{6} B_{\mu} B^{\mu}\right)\right) \\
& =\left(f_{k}^{2}\left(\partial_{\mu} h_{0}^{*} \partial^{\mu} h_{0}+\partial_{\mu} h_{1}^{*} \partial^{\mu} h_{1}\right)+f_{k}^{2}\left(h_{0} h_{0}^{*}+h_{1} h_{1}^{*}\right)\left(2 g^{2} W_{\mu}^{a} W^{\mu a}+g^{\prime 2} \frac{5}{6} B_{\mu} B^{\mu}\right)\right) \frac{\operatorname{Tr}\left(K^{\dagger} K\right)}{\lambda^{4}}
\end{aligned}
$$

The last term can be calculated as follows

$$
-\frac{\lambda^{2}}{f_{k}^{2} \lambda^{8}} \operatorname{Trace}\left(F_{55}^{\dagger} F_{55}\right)=-\frac{f_{k}^{2}}{2}\left(\bar{H} H-\frac{2 m^{2}}{f_{k}^{2}}\right)^{2} \operatorname{Trace}\left(K^{\dagger} K\right)^{2} .
$$

Finally, collecting the obtained terms we have

$$
L_{g}=-\frac{1}{f_{k}^{2}}\left(\frac{g^{2}}{4} \operatorname{Tr}\left(W_{\mu \nu}^{a} W^{\mu v a}\right)+g^{\prime 2} \frac{5}{6} B_{\mu \nu} B^{\mu v}\right)+2 D_{\mu} \bar{H} D^{\mu} H-\frac{f_{k}^{2}}{2}\left(\bar{H} H-\frac{2 m^{2}}{f_{k}^{2}}\right)^{2}
$$




\section{Constraints and Predictions}

In order to have the right factors for kinetic terms of the gauge fields, we imply that

$$
\begin{aligned}
& \frac{1}{f_{k}^{2}} \frac{g^{2}}{4}=\frac{1}{4} \rightarrow f_{k}=g \\
& \frac{1}{g^{2}} g^{\prime 2} \frac{5}{6}=\frac{1}{4} \rightarrow g^{\prime}=g \sqrt{\frac{3}{10}} .
\end{aligned}
$$

Hence the Weinberg angle is calculated explicitly as follows

$$
\sin ^{2} \theta_{W}=\frac{g^{\prime 2}}{g^{2}+g^{\prime 2}}=\frac{3}{13} \approx 0.23077
$$

The deviation of this prediction is just $0.1 \%$ compared to the experimental value.

In order to have the right factor for the kinetic term of the Higgs field, we have

$$
\operatorname{Tr}\left(K^{\dagger} K\right)=12 N_{f} m_{W}^{2} \sin ^{2} \theta_{W} /\left(2-\sin ^{2} \theta_{W}\right)
$$

Using the constraint (35) we obtain a prediction of the top quark mass

$$
m_{t}=173.45 \sim \mathrm{MeV},
$$

which is also in a very good agreement with experimental data of the Particle Data 2018 [19].

The Higgs mass can be calculated as

$$
m_{H}^{2}=2 m_{t}^{2} / \lambda^{2}
$$

If we choose the internal metric parameter $\lambda=2$, the Higgs mass is predicted as $122.65 \mathrm{MeV}$ in a good agreement with experiment with $1.8 \%$ deviation.

\section{Conclusions}

The geometric approach called DKKT to the construction of the spontaneously broken gauge theory is based on a discrete dimension having only two points. This approach can also be applied to constructed the extended Einstein's gravity, leading also to the nonabelian gauge vector fields as its components. So the construction procedure of the theories of gravity and Yang-Mills gauge fields is the same.

It is remarkable in the physical models based on the discrete dimensions, there are constraints on the model parameters not by symmetries. These lead to the predictions, whose verifications can confirm the new concepts of space-tume.

In this paper, we reexamined the predictions of the top quark and Higgs masses by Viet $[15,16]$ with new Dirac operator, the Yukawa coupling matrices and a specific metric structure of the discrete space dimension. The predictions are in a perfect agreement with experimental data, meaning that the discrete dimensions do exist at the electroweak scale.

\section{Acknowledgement}

Thanks are also due to Nguyen Suan Han and Tran Minh Hieu for their helpful discussions and supports. The research is funded by Vietnam National Foundation for Science and Technology Development (NAFOSTED) under grant number 103.01-2017.319. 


\section{References}

[1] T.P. T. Kaluza, Zum unitätsproblem der physik, Sitzungsber. Preuss. Akad. Wiss. Berlin (Math. Phys.) (1921) (arXiv: 1803.08616) 966-972.

[2] O. Klein, Quantum theory and five-dimensional theory of relativity, 1926 Z. Phys. 37 (1987) 895.

[3] G. Landi, N.A. Viet, K.C. Wali, Gravity and electromagnetism in noncommutative geometry, Physics Letters B $326(1-2)$ (1994) 45-50.

[4] N.A. Viet, K.C. Wali, A discretized version of Kaluza-Klein theory with torsion and massive fields, International Journal of Modern Physics A 11 (13) (1996) 2403-2418.

[5] N.A. Viet, K.C. Wali, Noncommutative geometry and a discretized version of Kaluza-Klein theory with a finite field content, International Journal of Modern Physics A. 11(3) (1996): 533-551.

[6] N.A. Viet, K.C. Wali, Chiral spinors and gauge fields in noncommutative curved space-time, Physical Review D, 67(12) (2003) 124029.

[7] N.A. Viet, P.T. Du, Non-Abelian gauge fields as components of gravity in the discretized Kaluza-Klein theory. Modern Physics Letters A. 32(18) (2017) 1750095.

[8] N.A. Viet, N.V. Dat, N.S. Han, K.C. Wali, Einstein-Yang-Mills-Dirac systems from the discretized Kaluza-Klein theory. Physical Review D. 95(3) (2017) 035030.

[9] N.A. Viet, Talk given at Rencontres du Vietnam on "Cosmology-50 year after CMB discovery", Quy Nhon, Vietnam (2015).

[10] C. Deffayet, J. Mourad, Deconstruction of gravity, International Journal of Theoretical Physics, 44(10) (2015) 1743-1752.

[11] Alishahiha, Mohsen, (De) constructing dimensions and non-commutative geometry, Physics Letters B 517.3-4 (2001) 406-414.

[12] Arkani-Hamed, Nima, and Matthew D. Schwartz, Discrete gravitational dimensions, Physical Review D 69.10 (2004) 104001.

[13] A. Connes, J. Lott, Particle models and noncommutative geometry. Nucl. Phys. B, 18 (1991) 29-47.

[14] A.H. Chamseddine, G. Felder, J. Fröhlich, Gravity in non-commutative geometry. Communications in Mathematical Physics 155(1) (1993) 205-217.

[15] Nguyen Ai Viet, Predictions of noncommutative space-time, In: Talk given at. (1994) p. 0207-212.

[16] Nguyen Ai Viet, Discrete internal space and its visibility, Acta Physica Hungarica New Series Heavy Ion Physics 1.3-4 (1995) 263-272.

[17] Cheng, L.F. Li., Gauge theory of elementary particle physics, Clarendon Press, Oxford, 1984.

[18] Nguyen Ai Viet, Extra dimension of space-time exposed by anomalies at low energy, arXiv preprint arXiv:1907.04517 (2019).

[19] Tanabashi, Masaharu, et al, Review of particle physics, Physical Review D 98.3 (2018) 030001. 